\title{
Lidil
}

Revue de linguistique et de didactique des langues

$56 \mid 2017$

Grammaires et littéracies

\section{Le commentaire stylistique du CAPES : un exercice académique au service des compétences en littéracie?}

Stylistic Commentary on French CAPES: An Academic Exercise at the Service of Literacy Skills?

Corinne Delhay

\section{OpenEdition}

Journals

Édition électronique

URL : http://journals.openedition.org/lidil/4794

DOI : $10.4000 /$ lidil.4794

ISSN : $1960-6052$

Éditeur

UGA Éditions/Université Grenoble Alpes

Édition imprimée

ISBN : 978-2-37747-024-2

ISSN : $1146-6480$

Référence électronique

Corinne Delhay, « Le commentaire stylistique du CAPES : un exercice académique au service des compétences en littéracie ? », Lidil [En ligne], 56 | 2017, mis en ligne le 01 novembre 2017, consulté le 10 décembre 2020. URL : http://journals.openedition.org/lidil/4794; DOI : https://doi.org/10.4000/ lidil.4794

Ce document a été généré automatiquement le 10 décembre 2020.

(C) Lidil 


\title{
Le commentaire stylistique $\mathrm{du}$ CAPES : un exercice académique au service des compétences en littéracie?
}

\author{
Stylistic Commentary on French CAPES: An Academic Exercise at the Service of \\ Literacy Skills?
}

Corinne Delhay

\section{Introduction}

1 La littéracie, définie par l'OCDE comme «la capacité de comprendre, d'utiliser et d'analyser des textes écrits, afin de pouvoir réaliser ses objectifs, développer ses connaissances et son potentiel et jouer un rôle actif dans la société ${ }^{1}$ » est la résultante de diverses compétences qui sont à la fois cognitives, sociales et linguistiques (Moreau, 2013, p. 16). Les compétences linguistiques elles-mêmes peuvent se décliner en diverses compétences, aussi bien orthographique, lexicale, syntaxique, pragmatique ou rhétorique qui, ensemble, permettent l'accès à l'interprétation des textes. Mais, de même qu'il existe une grande diversité dans la complexité des textes, de même faut-il envisager des degrés dans les compétences scripturales. La notion de grammaire avancée renvoie à des compétences en grammaire de texte et de discours qui vont bien au-delà des strictes compétences normatives. Mais comment enseigner la grammaire avancée? Le champ relativement récent des littéracies universitaires qui recouvre, selon Lang et Meyer (2015, p. 224), «[...] l'apprentissage et la maitrise des discours en usage dans la formation académique, que celle-ci soit tournée vers l'axe professionnel, à travers les concours par exemple, ou vers l'axe scientifique, à travers la production de résultats d'investigation ", invite à s'intéresser à la didactique de la grammaire avancée pour autant qu'elle est indissociable de la maitrise de certains types d'écrits. 
2 Nous nous sommes intéressée à la formation des futurs enseignants de lettres. En effet, c'est aux professeurs de français qu'incombe majoritairement, dans le second degré, la responsabilité de faire acquérir au plus grand nombre les compétences en littéracie. Or, il existe dans la formation des enseignants de français un exercice académique très codifié, le commentaire dit stylistique du CAPES de lettres, qui a pour enjeu de mettre les compétences en matière de grammaire de phrase, de textes et de discours au service de l'interprétation du texte littéraire. Cet exercice offre un modèle idéal pour vérifier à la fois les compétences des futurs enseignants en matière de "grammaire avancée » mais aussi leur capacité métacognitive à expliciter ces compétences dans la relation didactique.

3 Notre travail part du postulat que les rapports de jury des concours de recrutement des professeurs de lettres définissent la méthodologie et le métalangage attendus pour cet exercice académique. Nous plaçant délibérément dans le cadre actuel de la formation des professeurs de lettres, nous nous sommes appuyée sur les rapports de jury de concours de 2011 à 2016 qui correspondent à la dernière modification du concours du CAPES de lettres. Notre travail se déploiera en trois temps. Dans une première partie, nous montrerons que le commentaire dit stylistique est pleinement une épreuve de linguistique qui permet d'accéder à l'interprétation de tout texte à partir de l'identification de faits langagiers. Nous rappellerons donc l'origine du commentaire stylistique, conçu comme étude grammaticale d'un texte en français. Dans une seconde partie, nous présenterons les résultats d'une enquête que nous avons réalisée auprès d'étudiants préparant le CAPES de lettres modernes afin de déterminer leur degré de familiarité avec un certain nombre d'items linguistiques les plus fréquemment cités dans les rapports de jury à propos du commentaire dit stylistique. Dans une troisième partie, nous essaierons de montrer que le commentaire stylistique pourrait servir de matrice pour l'évaluation générale des compétences en matière de grammaire avancée, et ainsi être transposé à l'ensemble des textes, qu'ils soient littéraires ou non.

\section{Du commentaire d'un texte en français au commentaire stylistique}

4 Depuis la dernière réforme du CAPES de lettres, en 2014, l'étude stylistique est une des quatre épreuves qui permettent, à égalité de points, d'évaluer la compétence des futurs professeurs de lettres au sein de l'épreuve intitulée "Étude grammaticale de textes de langue française ${ }^{2} »$. Nous ne discuterons pas ici du statut toujours problématique de la "stylistique» dans le champ de la «linguistique» (Bordas, 2007), mais nous nous contenterons de considérer l'existence du commentaire stylistique comme une donnée de fait au CAPES. Par sa place au sein des épreuves du CAPES, il semble incontestable ${ }^{3}$ que « la stylistique reste un travail sur la langue » (Bordas, 2007, p. 246), ce que confirme le rapport de jury de 2004 qui parle «d'un devoir de linguistique appliquée à un corpus littéraire ».

\subsection{Origine du commentaire dit stylistique}

5 Bordas (2007, p. 242) a montré que le commentaire stylistique, tel qu'il est conçu dans les concours de recrutement des professeurs, a été défini par Antoine (1959), fondateur, en 1960, de la nouvelle agrégation de lettres modernes: 
[...] Le stylisticien doit $1^{\circ}$ constituer le catalogue des procédés utilisés par l'artiste, i. e. les identifier par rapport à l'état de langue où ils ont vu le jour; $2^{\circ}$ les " expliquer », i. e. les motiver et les caractériser en fonction de l'intention qui les a fait choisir. Il faut seulement s'entendre, pour finir, sur les limites, la nature, les modalités de ces deux opérations. (p. 55)

Mais il a également établi (Bordas, 2003, p. 582) que c'est Lanson ${ }^{4}$ (1925) qui a voulu proposer un rééquilibrage dans la formation des professeurs de lettres en l'étayant sur deux piliers fondamentaux et complémentaires : l'histoire littéraire d'un côté, évaluée par l'exercice de composition française, l'étude de la langue d'autre part, évaluée par l'explication d'un texte en français.

L'exercice de l'explication a pour but, et, lorsqu'il est bien pratiqué, pour effet, de créer chez les étudiants une habitude de lire, attentivement, et d'interpréter, fidèlement, les textes littéraires. Il tend à les rendre capable de trouver dans une page ou une œuvre d'un écrivain ce qui y est, tout ce qui y est, rien que ce qui y est. (Lanson, 1925, p. 40)

En un mot, lire avec réflexion, lire pour comprendre et de façon à comprendre, lire pour se donner non seulement des impressions fortes ou des impressions multiples, mais pour acquérir une intelligence claire, précise et distincte des textes [...], c'est une chose qui ne se fait pas toute seule [...], c'est une chose qui s'apprend, et c'est la chose qu'on apprend par l'exercice de l'explication de texte. (Lanson, 1925, p. 44-45)

Par cette innovation, Lanson soulignait «l'importance de l'étude de la langue dans l'analyse méthodique d'un texte littéraire français " (cité par Bordas, 2003, p. 582), et affirmait la nécessité d'une double formation, à la fois linguistique et littéraire dans la formation des professeurs de lettres. Ce postulat reste à priori inchangé puisque les concours de recrutement comportent ces deux composantes, et elles sont tout particulièrement associées dans l'épreuve dite du « commentaire stylistique ».

\subsection{Métalangage et compétences attendues}

7 L'épreuve de stylistique est vue comme une épreuve "technique ", qui suppose une démarche méthodique et s'appuie sur un métalangage précis. La transposition didactique de notions linguistiques ne va pas de soi, et les rapports de jury des concours de recrutement des professeurs de lettres peuvent être considérés comme révélateurs des "avancées» théoriques - éventuellement critiquables - jugées suffisamment consensuelles pour être transposées dans l'enseignement. À la lecture des rapports récents (2011-2016) ${ }^{5}$ du jury du CAPES de lettres modernes, nous avons tenté de circonscrire le champ des notions linguistiques qui sont supposées connues des candidats.

Les rapports fournissent souvent « une liste commentée des postes stylistiques attendus » (R13, p. 65), voire « l'ensemble des postes d'analyse stylistique qui étaient attendus par le jury » (R14exc, p. 79). Le relevé systématique des termes métalinguistiques présents dans les sept rapports analysés nous donne deux indications essentielles. Tout d'abord, on peut affirmer que les «postes d'analyse stylistiques » coïncident quasiment avec toutes les entrées de la Terminologie grammaticale de 1997. On y trouve en effet, outre des termes généraux désignant des unités ou des fonctionnements linguistiques, le vocabulaire de la grammaire de discours, celui de la grammaire textuelle et celui de la grammaire de phrase ainsi que le vocabulaire de la lexicologie dans ses aspects formels et sémantiques.

9 Ainsi, les Notions générales qui ouvrent la Terminologie grammaticale sont exploitées dans les rapports au titre des connotations (R11, 14exc), de l'oralité (R11, R12, R14exc), ou des questions de rythme (R11, R12, R13, R14ord). La grammaire de discours est largement 
présente : dispositif énonciatif (R11, R12, R14ord), commentaires métadiscursifs (R14ord, $R 16)$, plans d'énonciation, situation d'énonciation (R12, R13), valeurs des temps (R11, R12, R13, R14exc, R15, R16), discours rapporté (R11, R12, R14exc), modalités et/ou modalisateurs (R11, R12, R13, R14, R15), actes de parole (R13, R14ord, R15) et contenus implicites (R14ord, R15), etc. La question des genres littéraires apparait derrière le titre de Types de discours, décliné en genre théâtral (R14ord), genre autobiographique (R12), genre narratif (R16), genre épidictique (R13), genre poétique (R15), genre argumentatif (R13), tandis que la question des registres littéraires (burlesque, comique, parodique...) est évidemment absente de la Terminologie. Les notions de grammaire de texte sont également évoquées dans les rapports de jury : thème/propos à travers la question de la thématisation (R12, R14exc, R16) ou de la progression thématique (R11, R12, R13, R14ord); modes de désignation (R11, R12, R14exc, R14ord, R15, R16) et chaine de référence (R16), à travers les questions d'anaphore (R11, R16); cohésion et cohérence à travers les connecteurs (R11, R12, R13, R14exc, R14ord, R16). Enfin, la grammaire de phrase est présente à travers des analyses de l'organisation syntaxique (R11, R12, R13, R14exc, R15), des types (R11, R12, R14ord) et formes (R11, R12, R13, R14ord) de phrase, des classes de mots ou des fonctions dans la phrase.

Les rapports de jury nous indiquent également les domaines non strictement linguistiques qui sont attendus. Il s'agit du vocabulaire de la narratologie ou de l'analyse de discours et surtout du vocabulaire de la rhétorique, essentiellement figurative. Il faut mettre à part les trois grands tropes (métaphore, métonymie, synecdoque), cités dans la Terminologie de 1997, qui sont situées à l'interface entre rhétorique et linguistique, et susceptibles d'une description sémantico-syntaxique souvent riche sur le plan interprétatif. En dehors de ces figures, il y a une abondance de termes rhétoriques dans les rapports de jury, ce qui peut être considéré comme une preuve de la « revanche » ou du retour subreptice de la rhétorique que Bordas (2003, p. 580) a mis en évidence. Les rapports de jury renvoient également au vocabulaire de la versification. Enfin, grâce aux ouvrages de C. Kerbrat-Orecchioni (1990-1992-1994, 2001) en particulier, le vocabulaire de la pragmatique (maximes conversationnelles, enchainement des répliques (R14ord)) et des interactions verbales (dimension verbale / kinésique / proxémique (R14ord)) est considéré comme un prérequis de la compétence du futur professeur de français.

\subsection{Petit recul critique : polyphonie ou cacophonie?}

11 Bien évidemment, les textes proposés au concours sont fort différents les uns des autres et les rédacteurs des rapports de jury émanent de divers corps de l'Éducation nationale. Néanmoins, les étudiants lecteurs de ces rapports sont souvent déstabilisés par certaines discordances que la juxtaposition des documents amplifie encore. Ainsi, l'utilisation du métalangage linguistique tend à perdre sa rigueur scientifique pour se dégrader en formules plus approximatives. Prenons l'incontournable question de l'étude des systèmes énonciatifs dans le texte: parle-t-on de plan du discours (R14exc) ou de régime de discours (R13) ? de grammaire de l'énonciation (R14ord) ou de domaine de l'énonciation (R16) ? de plan du récit (R14exc), ou de modalités de l'énonciation narrative (R16)? L'emploi du terme modalité, parfaitement intelligible pour un expert est souvent source de confusions pour les étudiants qui ont du mal à percevoir que les modalités d'énonciation évoquées dans un rapport (R11, R14ord) sont parfois appelées modalités phrastiques (R11) et qu'elles correspondent ailleurs aux types de phrase (R11, R12, R14ord, R15) des programmes scolaires. De même, si les étudiants ont l'obligation de bien 
connaitre les programmes pour la question de mise en situation professionnelle, il n'est sans doute pas indifférent de leur parler de forme impersonnelle (R11) plutôt que de tour impersonnel (R11) ou de tournures impersonnelles (R12), même si ces variantes peuvent sembler de peu d'importance.

Les candidats au professorat de lettres doivent maitriser un métalangage linguistique précis, mais aussi en phase avec les instructions officielles qu'ils seront appelés à connaitre dans leur futur métier. Mais qu'en est-il de leurs compétences linguistiques à l'entrée en master?

\section{Enquête sur les compétences des étudiants en matière de linguistique}

\subsection{Description et protocole de l'enquête}

13 Nous avons soumis, au cours du premier semestre de leur formation, un questionnaire à 37 étudiants de première année du master « Métiers de l'enseignement, de l'éducation et de la formation, Second degré, Enseigner les lettres", autrement dit des étudiants préparant le CAPES de lettres modernes ou de lettres classiques (voir annexe 1). Ce questionnaire demandait aux étudiants d'indiquer, selon une échelle allant de 0 à 4 , leur degré de familiarité avec 70 items correspondant à des notions, généralement linguistiques, utilisées dans les commentaires stylistiques des rapports de jury du concours de lettres modernes entre 2011 et 2016 (voir annexe 2, classement alphabétique). Ces termes correspondent aux grandes sections de la Terminologie grammaticale de 1997 et ont aussi été choisis pour correspondre aux besoins métalinguistiques induits par les épreuves actuelles du CAPES de lettres, à savoir la relation graphie/phonie, la lexicologie, la morphosyntaxe, et la stylistique. En voici une présentation classée selon les domaines qui apparaissent dans la Terminologie de 1997 :

Notions générales : graphème, phonème.

Grammaire de discours (dont pragmatique) : genres littéraires, registres littéraires, modalités d'énonciation, déictique, énonciation de discours, énonciation de récit, discours rapporté, texte argumentatif, descriptif, valeurs des temps de l'indicatif, aspect, types de texte, marques de subjectivité, axiologique, acte de langage, implicite, présupposé, sous-entendu, valeur illocutoire.

Grammaire de texte : cohésion textuelle, progression textuelle, chaine de référence, connecteurs, anaphore linguistique, modes de désignation, présentatif, thématisation, référence générique, référence indéfinie.

Grammaire de phrase : phrase de base, phrase atypique, phrase averbale, types et formes de phrase, phrase simple, parataxe/hypotaxe, conjonction de coordination, mot subordonnant, subordonnée relative, subordonnée complétive, subordonnée conjonctive, subordonnée interrogative, subordonnée circonstancielle, classes de mots, préposition, pronom, adjectif, adverbes, déterminants, nom, types de verbe, sujet (grammatical), attribut, complément de phrase, complément de verbe.

Lexicologie: lexie, polysémie, antonymie, autonymie, hyperonymie/hyponymie, synonymie, champ lexical, isotopie, métaphore, métonymie, synecdoque.

Nous avons exclu de la liste les termes relevant de la versification et de la rhétorique des figures, à l'exclusion des trois grands tropes (métaphore, métonymie, synecdoque). Les items retenus ont été présentés de façon alphabétique, ce qui a permis de masquer les proximités théoriques (p. ex. Implicite, Présupposé et Sous-entendu). L'exploitation, strictement indicative, des réponses à ce questionnaire est censée nous apporter deux 
types d'informations. Tout d'abord, il peut nous indiquer le degré de familiarité que les étudiants déclarent avoir des notions linguistiques. Le deuxième enseignement, corolaire du premier, est de révéler des zones plus fragiles que d'autres qui peuvent être source d'un sentiment d'insécurité linguistique.

\subsection{Présentation et discussion des résultats}

Si l'on observe la forme de la courbe correspondant à la moyenne des réponses enregistrées (voir fig. 1 ci-dessous), on constate que peu de notions sont déclarées inconnues ou très mal connues.

Figure 1. - Courbe des scores moyens par item (avec affichage de la moyenne générale).

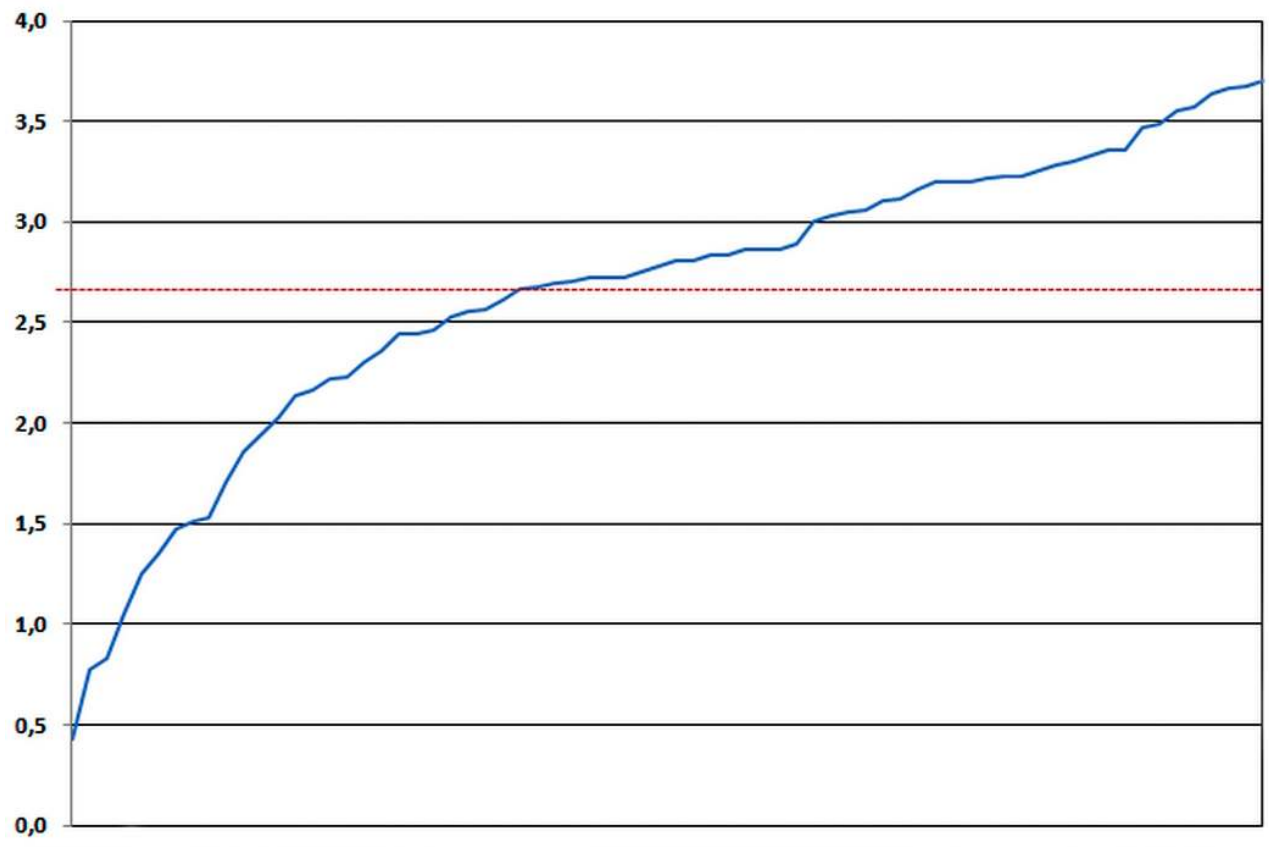

16 Cependant, l'analyse des réponses (voir annexe 2, classement par score) montre que les étudiants entrant en master MEEF déclarent ignorer ou mal connaitre certains items, avec une répartition des réponses différente dans les quatre domaines établis. Pour vérifier ce constat, nous nous sommes appuyée sur dix items particulièrement représentatifs des quatre domaines concernés, afin de tenter une forme de comparaison entre les quatre domaines.

L'annexe 3 présente la liste des 40 items retenus dans les quatre domaines, ainsi que le score moyen de familiarité déclarée pour chaque item. On observe ainsi que le domaine de la grammaire de texte présente un score moyen de 2,070 , celui de la grammaire de discours de 2,328, celui de la lexicologie de 2,555 et celui de la grammaire de phrase de $3,123^{6}$. 
Figure 2. - Courbes comparées des items regroupés par domaine.

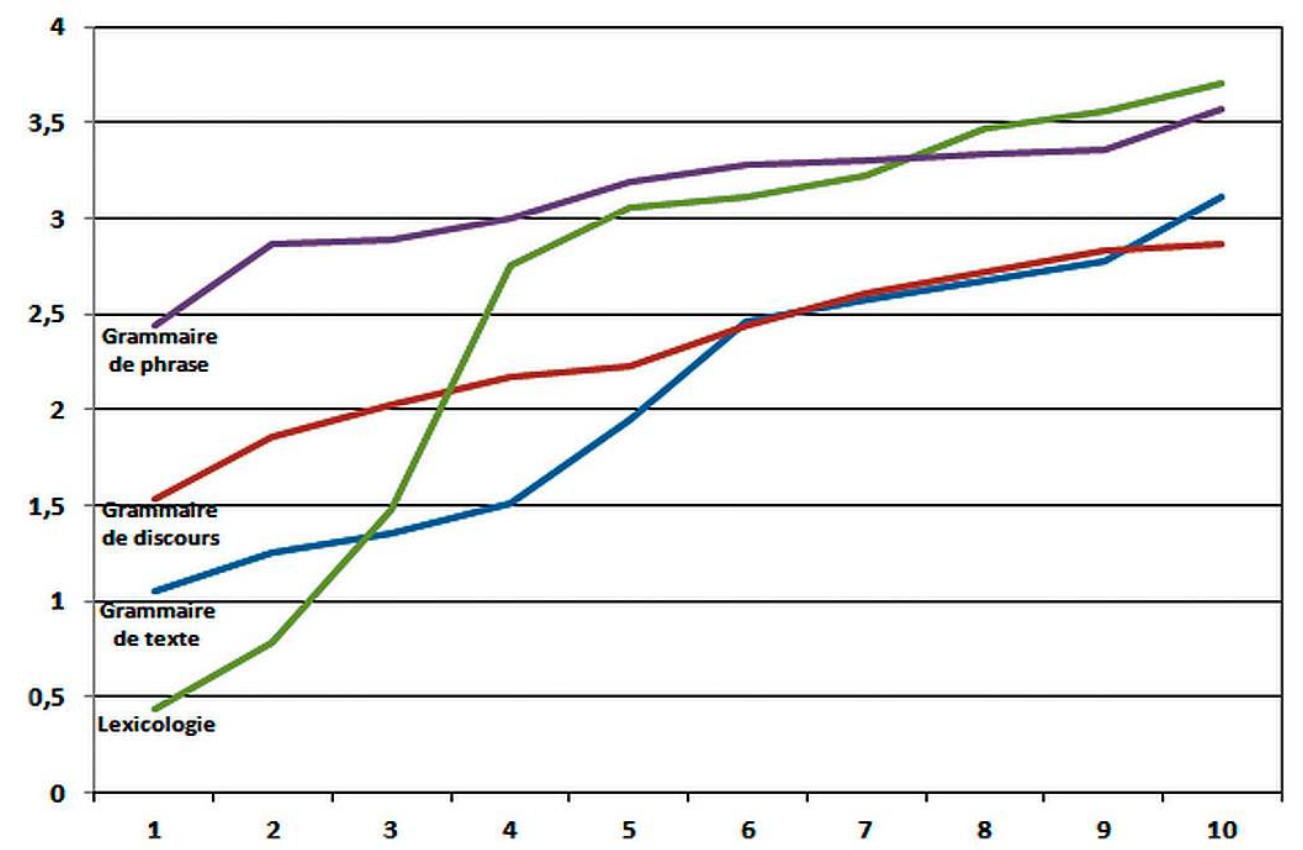

Parmi les items qui relèvent de l'analyse textuelle au sens large, on constate, en examinant l'ensemble des items du questionnaire, que les termes qui figurent dans les programmes du lycée (argumentatif, descriptif, types de textes, connecteurs) sont déclarés mieux connus que les termes appartenant plus particulièrement à l'enseignement universitaire (mode de désignation, chaine de référence), et cela même quand il ne s'agit souvent que d'infimes nuances terminologiques. Ainsi, le terme axiologique n'obtient qu'un score de 1,703/4 quand le terme englobant marques de subjectivité obtient un score de $2,861 / 4$. Une autre zone de fragilité semble être le domaine de la pragmatique au sens d'étude des interactions verbales. Près de la moitié des étudiants ne donnent qu'un indice de familiarité de 0 ou de 1 à ces items. Même si l'item implicite recueille un assez bon score $(2,722 / 4)$, l'analyse des réponses fournies montre que le sens technique de présupposé $(1,861 / 4)$ et de sous-entendu $(2,444 / 4)$ est généralement mal connu. Si les étudiants pensent connaitre la notion d'acte de langage (score déclaré de 2,027/4), ils ne semblent pas tous l'associer avec la notion de valeur illocutoire, qui obtient un score bien inférieur (score de familiarité de 1,528/4). Les items correspondant à la sémantique lexicale se situent aux deux extrêmes de l'échelle, la notion d'autonymie semblant problématique pour la majorité (score de $0,432 / 4$ ), tandis que la notion de champ lexical - régulièrement présente dans l'enseignement secondaire - culmine à 3,703/4 de score de familiarité. La tendance générale qui ressort donc du classement des items traduit un niveau de maitrise du métalangage assez contrasté, avec une dichotomie entre, d'une part, une relative confiance pour des notions relevant de la grammaire de phrase et de la lexicologie et, d'autre part, une relative insécurité pour des notions de grammaire de texte ou de discours. Il est assez significatif que l'on puisse observer une répartition quasi binaire des 70 items proposés de part et d'autre du score de 2,65/4 avec d'un côté des items correspondant à la grammaire de texte et à la pragmatique et de l'autre des items considérés comme plus familiers, qu'ils relèvent de l'analyse syntaxique traditionnelle au collège ou de l'étude des procédés langagiers pratiquée au lycée. 
Bien évidemment, il n'y a pas de stricte corrélation entre l'idée que les étudiants se font de leurs compétences et leurs compétences effectives, du moins telles qu'elles peuvent être approchées à travers l'évaluation d'un devoir noté. Nous prendrons comme premier exemple la question de l'identification de classes de mots. Dans un devoir, nous avons demandé d'identifier un déterminant, un pronom, un adjectif et un adverbe. Le questionnaire " indice de familiarité » comportait lui aussi les items déterminant, pronom, adjectif et adverbe. L'évaluation chiffrée des 4 classes dans un texte donne un taux moyen de réussite de 2,92/4, alors que l'indice de familiarité déclaré est de 3,37/4. On observe également des distorsions plus importantes au niveau individuel : un étudiant qui évalue sa «compétence» à 3,75/4 n'obtient qu'une note de 1,5/4 à l'exercice et semble confondre, par exemple, préposition et pronom. Inversement un étudiant qui s'attribue un score de $3 / 4$ obtient $4 / 4$ à la même question. Si l'on prend le domaine de la phrase complexe, on observe les mêmes distorsions. Ainsi, les étudiants s'attribuent un score de familiarité de 3,64/4 pour l'identification des phrases simples, alors que le score de réussite réel n'est que de $3 / 4$ : les résultats de l'évaluation suggèrent chez les sujets une confusion entre phrase simple et phrase minimale, et une méconnaissance générale des notions_de phrases de base et de phrases atypiques. Il en va de même pour l'identification des relatives : alors que leur score de familiarité est de 3,36/4, leur score de réussite réelle n'est que de 2,55/4, du fait, semble-t-il, d'une confusion entre conjonctive complétive en que et relative. Encore le devoir ne portait-il que sur des relatives adjectives, fort peu problématiques. Enfin, le score de familiarité pour l'identification des subordonnées circonstancielles est de 2,86/4, alors que le résultat obtenu dans l'évaluation chiffrée n'est que de $1,68 / 4$.

$\mathrm{Si}$ « l'étude stylistique consiste à rendre compte de faits linguistiques et de procédés langagiers qui contribuent à la construction du sens d'un texte » (R16, p. 98), les étudiants ont besoin d'identifier et de nommer les phénomènes linguistiques lorsqu'ils doivent rédiger un commentaire stylistique. Pourtant, force est de constater qu'à leur entrée en formation, beaucoup d'étudiants préparant le CAPES rencontrent encore des difficultés à s'approprier le métalangage.

\section{Le commentaire « stylistique » : un modèle pour les littéracies universitaires?}

\subsection{Le commentaire stylistique : un outil dans la formation linguistique du professeur de français}

Nous avons vu que l'étude stylistique du CAPES a été conçue pour évaluer les compétences linguistiques des aspirants professeurs dans une épreuve, qui se veut le pendant de l'épreuve de composition française, selon le modèle voulu par Lanson (cf. supra 2.1). En effet, on peut considérer que le commentaire stylistique "à la française " est directement en lien avec les missions d'enseignement du professeur de français. Qu'on en juge à la lecture de cet extrait des récents Programmes (2015, cycle 4, p. 244-245), dans lesquels nous avons souligné tous les items qui définissent une compétence en grammaire avancée telle qu'elle figure dans la Terminologie de 1997 :

Construire les notions permettant l'analyse et la production des textes et des

discours.

Observation de la variété des possibilités offertes par la langue. 
Repérage de ce qui détermine un registre [...], et de ce qui le caractérise ( organisation du propos, lexique, syntaxe) [...].

Approche de la variation [...] en fonction du lieu, du contexte, du moyen de communication.

Prise en compte des caractéristiques des textes lus ou à produire.

Identification et interprétation des éléments de la situation d'énonciation : [...] ( marques de personne, de lieu et de temps) ; [...]

Observation, reconnaissance et utilisation de paroles rapportées, directement ou indirectement ; repérage des indices qui signalent le doute, ou la certitude.

Repérage et interprétation des marques de modalisation [...].

Identification et utilisation des éléments linguistiques de cohésion textuelle $: \underline{s}$ ubstituts nominaux et pronoms de reprise; procédés de désignation et de caractérisation, rôle des déterminants indéfinis et définis; indicateurs de temps et de lieu, de relations logiques; système des temps.

Identification des phénomènes orthographiques, en particulier incidences orthographiques sur les pronoms de reprise sujet et complément (il, elle, leur).

Identification et utilisation des marques d'organisation du texte (mise en page, typographie, ponctuation, connecteurs).

Observation de la progression thématique du texte.

Reconnaissance des formes actives/passives et de leurs valeurs sémantiques; permutations pour marquer l'insistance ou l'emphase; présentatifs; valeur sémantique de la phrase impersonnelle.

Comme on le voit, le métalangage de cet extrait des Programmes peut être directement mis en relation avec celui que nous avons dégagé de la lecture des rapports de jury. Cela indique-que non seulement l'exercice scolaire du commentaire littéraire, mais aussi celui - plus récent - de la lecture analytique, s'appuient sur des compétences linguistiques que les professeurs de français doivent maitriser pour pouvoir les transmettre à leurs élèves.

L'exercice de la lecture analytique, apparu dans les Programmes de $2008^{7}$, et redéfini dans les Programmes du lycée de 2010 et dans ceux du collège de 2015, déconcerte souvent les enseignants. Pourtant, comme l'étude stylistique, la lecture analytique est définie comme lecture interprétative à partir de ce que dit le texte :

[...] S'assurer de la compréhension des textes en confrontant ce que les élèves en disent à ce qui est écrit [...]. Il s'agit d'apprendre aux élèves à questionner euxmêmes les textes, non à répondre à des questionnaires qui baliseraient pour eux la lecture. Il est possible d'entrer également dans la lecture par un questionnement. (2008, p. 67)

24 L'enjeu déclaré de cet exercice est de mettre l'élève en position de récepteur principal de l'œuvre. Partant des impressions de lecture qu'il a recueillies auprès des élèves, le professeur de français est censé orienter les «sujets-lecteurs » vers les outils d'analyse qui vont leur permettre de valider ou d'invalider leurs hypothèses. Les écueils de la lecture analytique sont d'une part la paraphrase et d'autre part les erreurs d'interprétation produites par une prise en compte insuffisante des indices textuels. Or, selon Cazorla (2012), « analyser des indices textuels demande d'être capable de connaitre et de repérer certains faits de langue, avant même de pouvoir les analyser » (p. 4). On en revient ainsi toujours à la nécessité de donner aux élèves les outils pour identifier les faits de langue qui, selon nous, donnent accès au sens du texte. Nous trouvons dans ce numéro une illustration de cette méthode d'analyse dans l'article de Chnane-Davin et Cuq, qui montre comment l'accès au sens d'un texte littéraire (Matteo Falcone de Mérimée) peut se faire via les outils linguistiques. Ici, l'étude des types de phrase et des actes de parole qui y sont associés permet effectivement aux élèves d'accéder très finement au sens du texte 
tout en évitant la paraphrase. Preuve, s'il en est, que les enseignants de français peuvent aider leurs élèves à devenir des lecteurs autonomes en mettant à leur disposition des outils linguistiques pour construire leur interprétation du texte de façon raisonnée.

\subsection{Un défi pour l'avenir : commenter des textes appartenant à tous les genres}

Amener les élèves à maitriser la diversité des textes et des discours est un objectif partagé par tous les enseignants de français aussi bien au niveau primaire que secondaire, en FLM qu'en FLE. Les compétences en littéracie passent par la maitrise d'une réelle grammaire avancée. Mais l'évaluation des compétences discursives est bien plus difficile à effectuer que le simple contrôle orthographique ou syntaxique. Par exemple, beaucoup d'exercices de grammaire dite avancée en FLE ne portent que sur ces aspects-là, souvent dans une approche puriste. Ainsi, un exercice de test de français avancé 8 (niveau C1) propose un QCM portant sur des choix orthographiques (la conversation que nous avons évoqué / évoquée / évoquez / évoquer a eu lieu le 19 mars 2006 ; la plupart des entreprises est / sont confrontée / confrontées / confronter à des problématiques de réduction des coûts [sic]), ou sur des choix lexicaux souvent très normatifs (Par contre / en revanche, je crois qu'y [sic] a un souci/ problème concernant les délais de livraisons). En revanche, l'exercice académique du commentaire dit stylistique, qui réalise les objectifs d'une réelle grammaire avancée, est transposable à d'autres types de texte.

En effet, le cadre grammatical du commentaire linguistique d'un texte littéraire est large, puisqu'il s'appuie à la fois sur des connaissances en grammaire de phrase, en grammaire textuelle et en grammaire de discours qui permettent aux futurs enseignants d'identifier et de dégager des faits langagiers intéressants. De plus, les connaissances acquises supposent la maitrise d'un métalangage précis, transmissible aux élèves pour leur donner un outil leur permettant de commenter le texte de manière autonome (lecture analytique) et sans paraphrase.

Nous retrouvons ici les préoccupations manifestées dans le domaine du FLE pour une pédagogie qui puisse former des apprenants plus actifs et capables de lire de façon critique. Ainsi Pellet (2010, p. 575) déplore que la variation de la métalangue soit encore trop absente des curricula de FLE : "Les jeunes enseignants manquent en particulier d'une métalangue leur permettant d'expliquer les points de grammaire dépendant du discours, ainsi que de la conscience du rôle des facteurs sociolinguistiques et pragmatiques sur les formes grammaticales. » (p. 575) Si le commentaire dit stylistique a un effet positif sur le développement de la capacité à lire de manière efficiente un texte, il joue également un rôle non négligeable dans l'apprentissage raisonné de l'écrit. Comme le disait déjà Lanson (1925, p. 45), si lire « est quelque chose qui s'apprend », écrire est aussi quelque chose qui s'apprend par le commentaire linguistique des textes. En mettant au jour les ressorts linguistiques d'un genre ou d'une forme textuelle, on donne aux apprenants les moyens de s'approprier ce matériau et d'intérioriser les mécanismes de construction du texte.

En d'autres termes, c'est à partir de méta-analyses de données textuelles que des compétences de lecture-écriture peuvent s'élaborer. Nous rejoignons le point de vue de Pellet (2010, p. 590) :

La littéracie permet ainsi de mettre en avant une conception du savoir au-delà de connaissances «domaniales » afin d'inclure la capacité à analyser et à réfléchir (à 
travers lecture et écriture) sur ces connaissances afin de pouvoir agir de façon critique. En d'autres termes, la littéracie met en jeu la notion de métacognition pour une appropriation des pratiques discursives.

\section{Conclusion}

Les littéracies universitaires ne peuvent pas être construites ex nihilo, mais, au contraire, doivent s'appuyer sur des compétences scripturales antérieures, acquises généralement dans le cadre scolaire. Au moment où l'ambition de l'École doit être de former des citoyens autonomes, capables d'esprit critique face à la profusion des textes auxquels ils sont confrontés, il est indispensable, selon nous, d'accroitre la place de la linguistique dans la formation des enseignants. En effet, les compétences en littéracie sont d'ordre éminemment langagier et les enseignants doivent être de réels spécialistes de l'étude de la langue dans toutes ses dimensions, et pas seulement orthographique ou syntaxique. Le commentaire dit stylistique s'avère un modèle qui a fait ses preuves en matière d'évaluation des compétences de grammaire avancée, parce qu'il s'appuie sur la prise en compte de la dimension textuelle des faits langagiers. Ce modèle peut être transposé à tout type de texte, quel que soit le genre auquel il appartient, parce qu'il dépasse l'approche parcellisée de "notions» déconnectées des discours. Les linguistes ont réellement une carte à jouer dans le domaine des littéracies qui sont devenues non seulement un enjeu de formation mais même un enjeu économique.

\section{BIBLIOGRAPHIE}

ANTOINE, Gérald. (1959). La stylistique française, sa définition, ses buts, ses méthodes. Revue de l'enseignement supérieur, 1, 42-60.

BARRÉ-De Miniac, Christine, Brissaud, Catherine \& Rispail, Marielle. (2004). La littéracie: conceptions théoriques et pratiques d'enseignement de la lecture-écriture. Paris : L'Harmattan. BoIssIÉRAS, Fabienne. (2010). Arrogante stylistique ? Dans L. Bougault \& J. Wulf (dir.), StylistiqueS (p. 55-62). Rennes : Presses universitaires de Rennes.

BORDAS, Éric. (2003). Stylistique et histoire littéraire. Revue d'histoire littéraire de la France, 103, 579-589.

BORDAS, Éric. (2007). La « stylistique des concours ». Pratiques, 135-136, 240-248.

CAZORLA, Séverine. (2012). Quelle transmission à l'élève en lecture analytique au secondaire? Quels moyens ? Communication présentée à la Biennale internationale de l'éducation, de la formation et des pratiques professionnelles (Paris, juillet 2012). Disponible en ligne sur <http://halshs.archivesouvertes.fr/halshs-00769816/> (consulté le 28 mai 2017).

CHNANE-DAvin, Fatima \& CUQ, Jean-Pierre. (2017). Outils linguistiques et entrée en littéracie chez les élèves allophones. Lidil, 56 (ici-même). 
KERBRAT-ORECCHIONI, Catherine. (1990-1992-1994). Les interactions verbales (t. 1-2-3). Paris : Armand Colin.

KERBRAT-ORECCHIONI, Catherine. (2001). Les actes de langage dans le discours. Théorie et fonctionnement Paris : Nathan.

LANSON, Gustave. (1925). Méthodes de l'histoire littéraire. Paris : Les Belles Lettres. Disponible en ligne sur <http://gallica.bnf.fr/ark:/12148/bpt6k114231j> (consulté le 28 mai 2017).

LANG, Élodie \& MEYER, Jean-Paul. (2015). Grammaire avancée et littéracies universitaires. Vos papiers sont-ils en règle ? Dans M. Vinaver-Kovič \& V. Stanojević (dir.), Les études françaises aujourd'hui. Pourquoi étudier la grammaire? Théories et pratiques (p. 223-242). Belgrade : Faculté de philologie.

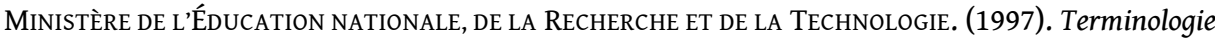

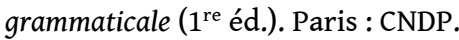

MoReau, André C. (2013). Le concept de littératie en francophonie : que disent les définitions ? CNRIS, 4(2), p. 14-18.

PelLet Stephanie. (2010). Critical Language Learning : le rôle de la littéracie et de la métacognition dans l'acquisition du français langue étrangère. Dans F. Neveu, T. V. Muni, J. Durand, T. Klingler, L. Mondada \& S. Prévost (éds), Actes du Congrès mondial de linguistique française (CMLF 2010) (p. 573-593). Paris : Institut de linguistique française. <http://dx.doi.org/10.1051/cmlf/2010183>.

\section{ANNEXES}

\section{Annexe 1. - Questionnaire}

Nom : Prénom : Date de naissance :

Date d'obtention du bac Série : Mention :

Études supérieures : Académie de Strasbourg : $\square$ Oui $\square$ Non $\square$ Autre (préciser) : Classe préparatoire (Lycée de) Licence : LM $\square$ LC $\square$ SDL $\square$ Autre :

Voici une liste de notions linguistiques pouvant être utilisées dans un commentaire stylistique.

a) Pour chaque item, indiquez votre degré de familiarité avec la notion (c'est-à-dire votre capacité à identifier sans erreur la notion dans le texte) à l'aide d'une « note » entre 0 et $4: 0$ (je ne connais pas) ; 1 (je connais mal) ; 2 (je connais un peu) ; 3 (je connais assez bien) ; 4 (je connais très bien).

b) Pour chaque item, donnez si possible un exemple.

\section{Annexe 2. - Résultats du questionnaire}




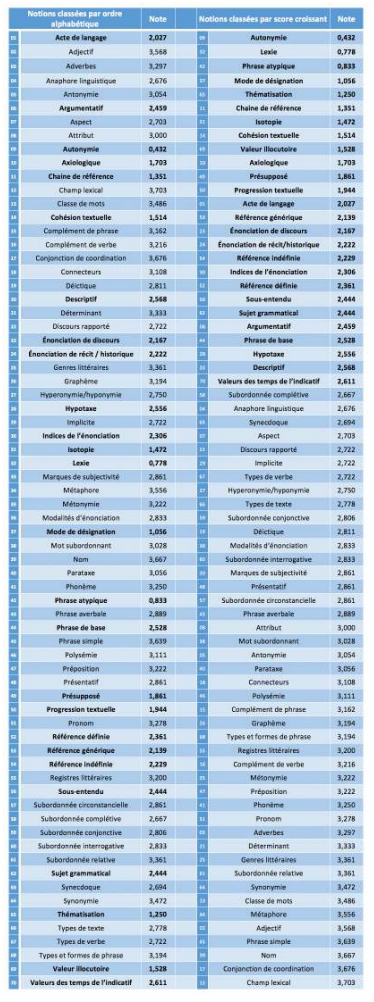

Les items et scores marqués en gras sont ceux situées en-dessous de la moyenne générale $(2,648)$.

Annexe 3. - Comparaison entre quatre sous-domaines à partir de la sélection de 10 items par domaine (GT : grammaire de texte ; GD : grammaire de discours ; LX : lexicologie ; GP : grammaire de phrase).

\begin{tabular}{|c|c|c|c|c|}
\hline 37 & GT & Mode de désignation & 1,056 & \multirow{10}{*}{2,070} \\
\hline 65 & GT & Thématisation & 1,250 & \\
\hline 11 & GT & Chaine de référence & 1,351 & \\
\hline 14 & GT & Cohésion textuelle & 1,514 & \\
\hline 50 & GT & Progression textuelle & 1,944 & \\
\hline 06 & GT & Argumentatif & 2,459 & \\
\hline 20 & GT & Descriptif & 2,568 & \\
\hline 04 & GT & Anaphore linguistique & 2,676 & \\
\hline 66 & GT & Types de textes & 2,778 & \\
\hline 18 & GT & Connecteurs & 3,108 & \\
\hline 69 & GD & Valeur illocutoire & 1,528 & \multirow{10}{*}{2,328} \\
\hline 49 & $G D$ & Présupposé & 1,861 & \\
\hline 01 & GD & Acte de langage & 2,027 & \\
\hline 23 & GD & Énonciation de discours & 2,167 & \\
\hline 24 & $G D$ & Énonciation de récit/historique & 2,222 & \\
\hline 56 & GD & Sous-entendu & 2,444 & \\
\hline 70 & $G D$ & Valeurs des temps de l'indicatif & 2,611 & \\
\hline 29 & $G D$ & Implicite & 2,722 & \\
\hline 36 & GD & Modalités d'énonciation & 2,833 & \\
\hline 33 & GD & Marques de subjectivité & 2,861 & \\
\hline 09 & Lx & Autonymie & 0,432 & \multirow{10}{*}{ 2,555 } \\
\hline 32 & $\mathrm{LX}$ & Lexie & 0,778 & \\
\hline 31 & $\mathrm{LX}$ & Isotopie & 1,472 & \\
\hline 27 & LX & Hyperonymie/hyponymie & 2,750 & \\
\hline 05 & $\mathrm{LX}$ & Antonymie & 3,054 & \\
\hline 46 & $\mathrm{LX}$ & Polysémie & 3,111 & \\
\hline 35 & $\mathrm{LX}$ & Métonymie & 3,222 & \\
\hline 64 & $\mathrm{LX}$ & Synonymie & 3,472 & \\
\hline 34 & $\mathrm{LX}$ & Métaphore & 3,556 & \\
\hline 12 & LX & Champ lexical & 3,703 & \\
\hline 62 & GP & Sujet grammatical & 2,444 & \multirow{10}{*}{3,123} \\
\hline 57 & GP & Subordonnée circonstancielle & 2,861 & \\
\hline 43 & GP & Phrase averbale & 2,889 & \\
\hline 08 & GP & Attribut & 3,000 & \\
\hline 68 & GP & Types et formes de phrase & 3,194 & \\
\hline 51 & GP & Pronom & 3,278 & \\
\hline 03 & GP & Adverbes & 3,297 & \\
\hline 21 & GP & Déterminant & 3,333 & \\
\hline 61 & GP & Subordonnée relative & 3,361 & \\
\hline 02 & GP & Adjectif & 3,568 & \\
\hline
\end{tabular}




\section{NOTES}

1. OCDE, Programme for International Student Assessment (PISA), 2000, p. 11.

2. La deuxième épreuve écrite, après la Composition française, est intitulée «Épreuve écrite à partie d'un dossier: étude grammaticale de textes de langue française». Elle dure 6 heures, comme l'épreuve de Composition française, et comporte une question d'« Histoire de la langue française » à partir d'un texte d'ancien français, une question d' "Étude synchronique du texte de français moderne ou contemporain " consistant en l'analyse du point de vue orthographique, lexicologique et grammatical d'un texte de français moderne ou contemporain, une question d'«Étude stylistique du texte de français moderne ou contemporain », une question de "Mise en perspective des savoirs grammaticaux", portant sur tout ou partie des documents du dossier. Chacune des quatre questions vaut 5 points sur 20.

3. Ce n'est pas le lieu ici de répondre à ceux qui, comme Boissiéras (2010, p. 55), contestent ce rattachement.

4. Lanson (1925, p. 57) : « Là, synthèse, analyse ici ; là, effort de création, ici, effort de critique ; là, développement de ce que l'on a en soi, ici, pénétration d'une pensée [...]. »

5. La référence à ces rapports sera effectuée sous forme abrégée, par exemple R14Exc pour le rapport du concours exceptionnel de 2014 ; R13, p. 65 pour un renvoi à la page 65 du rapport 2013.

6. Voir figure 2 et annexes 2 et 3.

7. Bulletin officiel $\mathrm{n}^{\circ} 6$, aout 2008 .

8. Voir <www.enseigna.fr>.

\section{RÉSUMÉS}

L'article s'intéresse à la didactique de la grammaire dite avancée (grammaire de texte et de discours) qui permet l'accès effectif à l'interprétation des textes.

Les recherches en littéracies universitaires invitent à travailler sur les méthodes permettant de développer les compétences en grammaire avancée chez les apprenants. C'est la raison pour laquelle l'auteure se penche sur un exercice académique très codifié de la formation des professeurs de lettres dont elle soutient qu'il pourrait servir de modèle à l'étude de n'importe quel type de texte. Les origines de cet exercice, le commentaire dit stylistique d'un texte littéraire, sont d'abord rappelées. Ensuite, à partir du métalangage présent dans les rapports récents du jury du CAPES de lettres modernes, sont présentés les résultats d'une enquête réalisée auprès d'étudiants préparant ce concours de recrutement. Un questionnaire détermine leur degré de familiarité avec différents items, présents dans la Terminologie grammaticale de 1997, et couvrant le champ de la grammaire de phrase, de texte et de discours. Le travail montre, à partir de graphiques, que les étudiants interrogés déclarent une relative familiarité avec les domaines de la grammaire de phrase et de la lexicologie et davantage d'insécurité linguistique avec la grammaire de texte et de discours. L'auteure défend l'idée que l'identification des faits de langue et la maitrise du métalangage est un indice de compétence en grammaire avancée. Elle soutient que l'exercice du commentaire stylistique est transposable à tout type de texte, car il donne une 
réelle maitrise du métalangage, permettant d'accéder aux mécanismes de la construction des textes et, ce faisant, d'améliorer les techniques d'écriture académique.

The article deals with the didactics of advanced grammar (text and discourse grammar), which gives effective access to the interpretation of texts.

Research in academic literacies calls for work on methods to develop advanced grammar skills in learners. The author examines therefore a highly codified academic exercise of the training of the professors of Letters, which she argues may serve as a model for the study of any type of text. The origins of this exercise, the so-called stylistic commentary on a literary text, are first recalled. Then, starting from the metalanguage presented in the recent reports of the Jury of the CAPES of French Language and Literature, the results of a survey are presented about students preparing this recruitment competition. A questionnaire determines their degree of familiarity with different items from the Terminology of Grammatic published in 1997, covering the fields of sentence, text and discourse grammar. The work shows that the students surveyed declare a relative familiarity with the areas of sentence grammar and lexicology and more linguistic insecurity with text and discourse grammar. The author defends the idea that the identification of the facts of language and the mastery of the metalanguage is an index of competence in advanced grammar. She maintains that the exercise of the stylistic commentary can be transposed to any type of text because it builds a real mastery of metalanguage, giving access to the mechanisms of the construction of texts and, in doing so, improving techniques of academic writing.

\section{INDEX}

Mots-clés : didactique, grammaire avancée, littéracies universitaires, degré de familiarité, insécurité linguistique, métalangage

Keywords : didactics, advanced grammar, academic literacies, level of familiarity, linguistic insecurity, metalanguage

\section{AUTEUR}

\section{CORINNE DELHAY}

Université de Strasbourg, laboratoire LiLPa 